\title{
Dexmedetomidine as Adjuvant Therapy for Acute Postoperative Neuropathic Pain Crisis
}

\author{
Thomas O’Neil, MD, Phillip E. Rodgers, MD, and Cameron Shultz, PhD, MSW
}

\begin{abstract}
Background: Dexmedetomidine is a potent $\alpha_{2}$-adrenergic agonist U.S. Food and Drug Administration (FDA) approved for sedation. While its use as an analgesic has been described in the palliative medicine literature, its use for managing an acute neuropathic pain episode is less well known.

Methods: Here we describe the use of adjuvant dexmedetomidine in a patient with metastatic sarcoma suffering from an acute postoperative neuropathic pain crisis.

Conclusion: Among patients with acute neuropathic pain for whom additional opioids raises respiratory-related concerns, the use of dexmedetomidine should be considered as a viable treatment alternative.
\end{abstract}

\section{Introduction}

D EXMedetomidine (Precedex ${ }^{\mathrm{TM}}$, Hospira, Lake Forest, IL) is an $\alpha_{2}$-adrenergic agonist approved by the U.S Food and Drug Administration (FDA) for sedation. ${ }^{1}$ Its unique mechanism of action allows it to be used in combination with opioids and benzodiazepines without concern for contributing to respiratory depression. ${ }^{1}$ In the palliative care literature, dexmedetomidine has been shown to benefit patients because it allows for interactive sedation at the end of life. ${ }^{2}$ Additional uses include treatment for complex regional pain syndrome, ${ }^{3}$ opioid-induced hyperalgesia, ${ }^{4}$ and intractable cancer pain. ${ }^{5}$ Although evidence shows that $\alpha_{2}$-agonists such as clonidine and dexmedetomidine can be helpful for acute pain management, they are less commonly used for this purpose. ${ }^{6}$ In this case study we discuss the use of dexmedetomidine in a patient on concurrent methadone therapy who experienced a postoperative neuropathic pain crisis unresponsive to high-dose opioids.

\section{Case Description}

G.B. is a 75-year-old man with a history of undifferentiated sarcoma of the left patella who was referred to the palliative care clinic by his primary care provider for treatment of longstanding nonmalignant back and knee pain related to his sarcoma. Over the previous year his primary care provider prescribed multiple opioid and nonopioid medications. G.B. was hospitalized in June 2011 for pain crisis of his left knee and was started on morphine patient-controlled analgesia (PCA). After dose finding, he was subsequently discharged on long-acting morphine, $60 \mathrm{mg}$ twice daily, and morphine immediate release (IR), $15 \mathrm{mg}$ daily. He was seen in followup in the palliative medicine clinic. At that time, due to ongoing pain, his long-acting morphine was increased to three times daily and morphine IR increased to $15-30 \mathrm{mg}$ four times per day as needed. A week later the patient called the clinic complaining of increasing pain, and long-acting morphine was increased to $120 \mathrm{mg}$ twice per day and morphine IR was increased to $30-45 \mathrm{mg}$ four times per day as needed. Three days later he called from home due to a pain crisis, fall, memory problems, hallucinations, and drowsiness. He was advised to go to the emergency department for further evaluation. Upon admission, palliative care consultants recommended rotation to methadone, $15 \mathrm{mg}$ orally, three times per day as needed, via the Morley-Makin protocol. ${ }^{7}$ Three days after discharge via phone visit, the patient reported that his pain was significantly improved and he was not experiencing any side effects. His methadone dosing schedule was $30 \mathrm{mg}$ orally every 8 hours, with $15 \mathrm{mg}$ orally every 4 hours as needed. Later that same day, the patient's son called to report that his father appeared somnolent, and methadone was decreased to $25 \mathrm{mg}$ orally every 8 hours and $10 \mathrm{mg}$ orally every 4 hours as needed.

Two days later G.B. was admitted to the hospital for a planned resection of sarcoma and lymph node dissection. His postoperative pain management course was complicated by a pain crisis that proved unresponsive to $400 \mu \mathrm{g}$ fentanyl intravenous push, $10 \mathrm{mg}$ intravenous morphine, $2 \mathrm{mg}$ intravenous midazolam, and $35 \mathrm{mg}$ methadone taken orally. Palliative care was consulted and the patient was admitted to the surgical intensive care unit (ICU) for fentanyl infusion, in addition to his oral methadone regimen. He responded well to $150 \mu \mathrm{g} / \mathrm{hr}$ 
infusion of fentanyl. Repeat operation and wound closure 1 week later had an uncomplicated postoperative course.

During recovery G.B. continued on methadone $30 \mathrm{mg}$ orally every 8 hours and $10 \mathrm{mg}$ orally every 4 hours as needed. His postoperative course, from the wound closure, was further complicated by poor wound healing requiring operative debridement 15 days after his initial resection. At that time, postoperative pain was managed with fentanyl infusion in the medical ICU. G.B. continued to have difficulty with wound healing, and required left above-the-knee amputation 14 days after debridement.

The palliative care team was paged to the postanesthesia care unit to address a pain crisis, where G.B. rated his pain at " 10 out of 10," describing it as "squeezing" and "vise-like" over his left leg. He had his eyes closed and was writhing on the bed during the examination. This pain was unresponsive to fentanyl infusion titrated to $350 \mu \mathrm{g} / \mathrm{hr}$. Despite his nothingby-mouth (NPO) status, he had not missed any methadone doses prior to his going to the operating room.

For G.B.'s refractory pain crisis, we recommended dexmedetomidine with a bolus of $0.5 \mu \mathrm{g} / \mathrm{kg}$ given over $20 \mathrm{~min}$ utes then $0.2 \mu \mathrm{g} / \mathrm{kg}$ per hour with the recommendation to increase by $0.2 \mu \mathrm{g} / \mathrm{kg}$ per hour every 30-60 minutes and titrate to analgesia, with a maximum dose of $1.5 \mu \mathrm{g} / \mathrm{kg}$ per hour. Overnight the dexmedetomidine was kept at $0.2 \mu \mathrm{g} / \mathrm{hr}$ and the fentanyl was reduced to $50 \mu \mathrm{g} / \mathrm{hr}$. The following day he reported his left leg pain at 8 of 10 but significantly improved. When seen on rounds he was able to speak and eat lunch. On postoperative day 2 he was weaned off both the fentanyl and dexmedetomidine infusions. Throughout his postoperative course he remained on methadone at previous dose of $30 \mathrm{mg}$ orally every 8 hours and $10 \mathrm{mg}$ orally every 4 hours as needed. Methadone was slowly decreased over the next few weeks due to decreased pain.

\section{Discussion}

Much of the literature describing the clinical application of dexmedetomidine emphasizes either its role in perioperative and intensive care sedation or its use in the management of routine postoperative pain as an opioid-sparing agent. ${ }^{8,9}$ This case study demonstrates the role that adjuvant dexmedetomidine can have in the management of an acute neuropathic pain crisis.

Despite high-dose fentanyl infusion while on concurrent methadone therapy for chronic pain, our patient had no significant relief of his postoperative suffering. G.B.'s postoperative consult with the palliative care team revealed he not only suffered from somatic postsurgical pain but also likely neuropathic phantom limb pain. The team was concerned that further titration of opioids or the addition of benzodiazepines could lead to respiratory depression-as experienced after a prior operation-hence warranting an agent with an alternative mechanism of action. Treatments such as ketamine and regional anesthesia were considered. However, because G.B. was already on methadone the effect of ketamine and its action on the $N$-methyl-D-aspartate receptor was likely to be minimal, and regional anesthesia was logistically unworkable due to staffing constraints and supply-related limitations.

Soon after the infusion of dexmedetomidine, G.B. not only obtained relief from his acute pain, he was able to get much needed rest. In sharp contrast to the previous evening, the morning after infusion he was able to eat and interact without concern for his pain. This rapid onset of pain relief makes dexmedetomidine an ideal agent for use during an acute neuropathic pain crisis. Similar to findings reported by Roberts et al. ${ }^{5}$ where dexmedetomidine was used to treat intractable cancer pain, G.B. was able to further taper the dose of methadone while maintaining good pain control. This suggests that patients using dexmedetomidine may benefit from its opioid dose-sparing effect, thereby limiting opioidrelated side effects. Importantly, while dexmedetomidine is a commonly used sedative, G.B. experienced neither excessive sedation nor somnolence while receiving the infusion, enabling him to actively participate in his own pain-management plan by accurately reporting his pain to the care team.

In addition to limiting opioid-related side effects, GB benefited from dexmedetomidine's relatively quick onset of action compared to agents more typically associated with the treatment of neuropathic pain. In this case, dexmedetomidine provided immediate relief of acute neuropathic pain that had been unresponsive to oral methadone and a high-dose fentanyl infusion. When considered in light of findings described by Nama et al., ${ }^{3}$ in which dexmedetomidine effectively relieved acute pain associated with complex regional pain syndrome, findings from this case underscore the utility dexmedetomidine may have in treating a number of refractory neuropathic pain syndromes.

Although dexmedetomidine is generally safe and well tolerated, cardiac side effects such as hypotension, bradycardia, and atrial fibrillation have been noted; ${ }^{1}$ however, an infusion rate of $0.2 \mu \mathrm{g} / \mathrm{kg}$ per hour was easily tolerated despite G.B.'s history of coronary artery disease.

While dexmedetomidine can be extremely effective in the management of an acute pain crisis, both its cost and a lack of familiarity or comfort with its use could be limiting in some settings. Likewise, because dexmedetomidine is not commonly used outside of operating rooms and postanesthesia care units, some clinicians may not feel comfortable with its use. Before using dexmedetomidine to treat acute pain, palliative care clinicians may need to establish unit-specific procedures, administration guidelines, and provide staff education.

\section{Conclusion}

Our patient, a 75-year-old man with metastatic sarcoma suffering from an acute episode of refractory postoperative neuropathic pain, experienced relief with the addition of dexmedetomidine to his medication regimen. This case further demonstrates the role that dexmedetomidine can play in the management of an acute pain crisis. Our patient not only experienced rapid relief from his pain-within hours of starting the dexmedetomidine infusion - there were neither reported nor observed side effects related to the therapy. While this case describes dexmedetomidine as a useful adjunct to traditional opioid medications, the relative absence of literature on the topic suggests dexmedetomidine may be underutilized in the palliative care setting. Among patients with acute neuropathic pain for whom additional opioids raises respiratory-related concerns, and the severity of pain requires an agent with a relatively rapid onset of action to control the neuropathic component of pain, the use of dexmedetomidine should be considered as a viable treatment alternative. 


\section{Acknowledgments}

The University of Michigan Institutional Review Board (UM-IRB) does not require its approval for clinical case studies involving one or two clinical experiences or observations identified in the course of clinical care, provided that the following FDA regulations requiring UM-IRB approval do not apply: articles (e.g., drugs, devices, biologics) that have not been approved for use in humans, articles requiring exemption from FDA oversight, and articles under an Investigational New Drug or Device Exemption. Likewise, the UM-IRB does not require its approval for standard practice, innovative care, or off-label use of FDA-approved drugs, biologics, devices, and other articles or substances that are used in the normal course of medical practice, provided the activity does not involve systematic collection of safety or efficacy data, and is limited to prevention, diagnosis, mitigation, treatment, or cure of disease in affected individuals.

\section{Author Disclosure Statement}

No competing financial interests exist.

\section{References}

1. Prommer E: Review article: Dexmedetomidine: Does it have potential in palliative medicine? Am J Hosp Palliat Care 2011; 28:276-283.

2. Soares LG, Naylor C, Martins MA, Peixoto G: Dexmedetomidine: A new option for intractable distress in the dying. J Pain Symptom Manage 2002;24:6-8.

3. Nama S, Meenan DR, Fritz WT: The use of sub-anesthetic intravenous ketamine and adjuvant dexmedetomidine when treating acute pain from CRPS. Pain Physician 2010;13:365368.

4. Belgrade M, Hall S: Dexmedetomidine infusion for the management of opioid-induced hyperalgesia. Pain Med 2010;11: 1819-1826.

5. Roberts SB, Wozencraft CP, Coyne PJ, Smith TJ: Dexmedetomidine as an adjuvant analgesic for intractable cancer pain. J Palliat Med 2011;14(3):371-373.

6. Chan AK, Cheung CW, Chong YK: Alpha-2 agonists in acute pain management. Expert Opin Pharmacother 2010;11: 2849-2868.

7. Morley JS, Makin MK: The use of methadone in cancer pain poorly responsive to other opioids. Pain Rev 1998;5: 51-58.

8. Lin TF, Yeh YC, Lin FS, et al.: Effect of combining dexmedetomidine and morphine for intravenous patient-controlled analgesia. Br J Anaesth 2009;102:117-122.

9. Wahlander S, Frumento RJ, Wagener G, et al.: A prospective, double-blind, randomized, placebo-controlled study of dexmedetomidine as an adjunct to epidural analgesia after thoracic surgery. J Cardiothorac Vasc Anesth 2005;19: 630-635.

Address correspondence to:

Thomas O'Neil, MD

Department of Family Medicine

University of Michigan Medical School 1150 West Medical Center Drive

M7300 Medical Science I SPC 5625 Ann Arbor, MI 48109

E-mail: thomasao@med.umich.edu 